\title{
Fc-Gamma Receptor III
}

National Cancer Institute

\section{Source}

National Cancer Institute. Fc-Gamma Receptor III. NCI Thesaurus. Code C17487.

A family of low affinity receptors that bind to the Fc region of gamma immunoglobulin (IgG) monomers and aggregates. These receptors are expressed on the surface of natural killer cells, neutrophils, monocytes and macrophages. Ig G binding initiates antibody-dependent cellular cytotoxicity and induces phagocytosis of antigen-antibody complexes. 